\title{
4. Der Krieg des Anastasius gegen die Isaurier
}

\subsection{Der Kriegsverlauf}

Nachdem Zeno am 9. April 491 gestorben war, wurde sein Nachfolger Anastasius am Gründonnerstag, den 11. April 491 gekrönt. Die konstitutionelle Frage, welche der gesellschaftlich mächtigen Gruppen, Heer, Senat oder das Kaiserhaus, denn nun eigentlich den Kaiser erhebt, war nun wieder die gleiche wie bei der Krönung des Marcian; letztlich bestimmte die Augusta, wer gekrönt wurde. ${ }^{1}$ Die für Isaurien be-

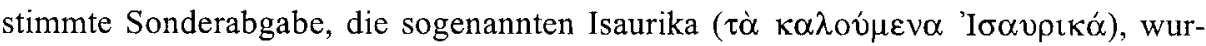
den unter Anastasius gleich nach seinem Regierungsantritt abgeschafft. ${ }^{2}$

Der Krieg gegen die Isaurier begann damit, daß nach Zenos Tod dessen isaurischer Bruder Longinus ungeachtet Anastasius' Krönung die Kaisermacht in isaurischen Händen halten wollte. ${ }^{3}$ Er war bereits Consul der Jahre 468 und 490 gewesen und stützte sich auf die in der Stadt ansässigen Isaurier und seinen Namensvetters Longinus von Cardala, der sich im Amt des magister officiorum befand. ${ }^{4}$ Aber ,,man hatte die Isaurier satt" in Konstantinopel. ${ }^{5}$ Gegen den Widerstand sowohl der nun

1 Theod. Lect. II 7; Theoph. AM 5983; Mal. XVI p. 392; Euagr. h.e. III 29; Mich. Syr. p. 18 , 6; De caer. I 92, p. 417-426; vgl. LILIE (1995): Der Bericht über die Krönung in De caer. I 92, p. 417-426 stammt höchstwahrscheinlich aus der Feder des Petrus Patricius aufgrund der Betonung der Rechtgläubigkeit des kommenden Kaisers und der Darstellung Ariadne als Werkzeug der Senatoren. Der praepositus sacri cubiculi Urbicius schlug vor, die Entscheidung der Kaiserin zu überlassen. Als sie Anastasius auswählte, freuten sich alle. Dies deutet vielmehr auf eine Initiative der Ariadne hin. Jedoch hatte sich der Senat zuvor selbständig versammelt, war also nicht von der Augusta einberufen worden. Volk und Heer werden von der Quelle zwar als weitere Entscheidungsträger genannt, spielen jedoch de facto keine Rolle. Man kann somit keineswegs von einer ,protokollarischen Genauigkeit“ des Zeremonienbuchs sprechen. Die Betonung der Rechtgläubigkeit des Anastasius im Protokoll ergibt keinen Sinn, da ein Aspirant für den Kaiserthron selbstverständlich rechtgläubig zu sein hatte, sowie die Aussage, daß das Volk einen wenig geizigen Kaiser sich gewünscht habe, ist aus der späteren Finanzpolitik des Anastasius zu erklären. In das Protokoll der Krönung sind somit anastasius-feindliche Elemente durch Petrus Patricius mit eingeflossen.

2 Als Motiv für den Isaurierhaß des Anastasius nimmt SchwarTz (1934) 216 an, daß er während seines langen Dienstes am Hof Gelegenheit gehabt hätte, ,die wüsten Gesellen kennenzulernen".

3 Marc. Com. s.a. 492; Mal. XVI p. 320 ThURN; Theoph. AM. 5983 ff.; Joh. Ant. frg. 100, frg. 214b; Jord. Rom. 354; Jos. Styl. 23; Euagr. h. e. III 29. 35; Theod. Lect. II 9; Zach. Rhet. h.e. VII 1 p. 102. Darstellung bei STEIN (1949) 82 ff.; SCHWARTZ (1934) $216 \mathrm{ff}$.

4 PLRE II 688 ff. s.n. 'Longinus 3 u. 6'. Euagr. h.e. III 29 u. 35 verwechselt Longinus von Cardala mit dem Zenobruder.

5 SChWARTZ (1934) 216. 
wieder zu Macht gekommenen senatorischen Opposition und auch der Stadtbevölkerung Konstantinopels, die ihren Antipathien bereits vor Zenos Regierungsantritt und während der Usurpationen von Basiliscus und Marcian Luft gemacht hatte, waren sie letztendlich machtlos. Der Versuch scheiterte; die Isaurier wurden aus der Stadt vertrieben. Dabei herrschten wiederum bürgerkriegsartige Zustände. Anlaß dazu gab ein Aufstand der Zirkusparteien, den angeblich die Isaurier angestiftet hatten; ${ }^{6}$ eine Propagandalüge ist hier naheliegend. Anastasius ging zunächst milde gegen die Isaurier vor. Er gestattete Longinus von Cardala, sich nach Isaurien zurückzuziehen. ${ }^{7}$ Auf die Nachrichten, daß dieser jedoch einen Aufstand vorbereite, konfiszierte Anastasius das Vermögen der in der Stadt ansässigen Isaurier. Longinus sammelte nun seine isaurischen Landsleute um sich, ${ }^{8}$ fiel plündernd in die Nachbarprovinzen ein und wandte sich mit einem veritablen Heerhaufen schließlich nach Konstantinopel. Auf dem Weg dorthin wurden sie bei Kotyaion in Phrygien (heute Kütahya) im Herbst 492 vernichtend geschlagen. ${ }^{9}$ Die Armee des Anastasius bestand aus thrakischen, gotischen und hunnischen Kontingenten unter dem Kommando des magister militum praesentalis Johannes' des Buckligen und des magister militum per Orientem Johannes Skytha. ${ }^{10}$ Der comes Isauriae Lingis, der die Seite der Aufständischen gewählt hatte, wurde getötet, die Überlebenden flohen nach Isaurien. Longinus wurde nicht hingerichtet, denn man wollte $\mathrm{zu}$ diesem frühen Zeitpunkt keinen Isaurieraufstand provozieren, sondern zum Mönch geschoren und in die Thebais in Ägypten verbannt, wo er acht Jahre später starb. ${ }^{11}$ Longinus von Cardala wurde seines Postens enthoben und setzte den Krieg von Isaurien aus fort. ${ }^{12}$

Der Kleinkrieg zog sich bis 498 hin. ${ }^{13}$ Der comes Isauriae bis ins Jahr 491, Lilingis qui et Lingis, ein Halbbruder des Illus, war von seiner Basis Papirion aus in Revolte. ${ }^{14}$ Hinzu kamen der militärisch engagierte Ex-Bischof Konon von Apamea und zwei Athenodoroi, von denen der eine senatorischen Rang innehatte. ${ }^{15}$ Antiochia am Cragus wurde zur Versorgungsbasis der Aufständischen. Longinus von Selinus, ein

6 Joh. Ant. frg. 214b; vgl. Marc. Com. s.a. 491 u. 493 (Zirkusaufstände).

7 Euagr. h.e. III 29; vgl. SCHWARTZ (1934) 216.

8 Theoph. AM 5985; Theod. Lect. Epit. 2.

9 TIB 5,1 41; TIB 7, s.v. - Hierzu SchwarTz (1934) 217: „die Isaurier bewiesen wiederum, daß sie im offenen Krieg nicht zu brauchen waren".

10 P. Oxy. 1959; Eustath frg. 6 = Euagr. h.e. III 35; Proc. anec. VI 5; Mal. 393; Theod. Lect. Epit. 449; Theoph. AM 5985. 5986; PLRE II 617, s.n. 'Fl. Ioannes qui et Gibbus 93'.

11 Joh. Ant. frg. 214 b; Theoph. AM 5984; Zon. XIV 3,20.

12 Theoph. AM 5983; vgl. Williams / Friell (1999) I98f.

13 Marc. com. a. 497: bellumque Isauricum hoc sexto anno sedatum.

14 PLRE II 683f., s.n. 'Lilingis'.

15 Theoph. AM 5985; Joh. Ant. frg. 214b. 
weiterer isaurischer Mächtiger diesen Namens, versorgte die Isaurier von hier aus mit Nachschub. ${ }^{16}$

Claudiopolis in der fruchtbaren Ebene leistete noch Widerstand, konnte jedoch 493 von dem comes scholarum Diogenes oder Diogenianus eingenommen werden, einem Verwandten der Augusta Ariadne. ${ }^{17}$ Bei der Schlacht von Kotyaion war er ein Offizier der Gardetruppen gewesen. ${ }^{18}$ Er wurde dann jedoch seinerseits von den Isauriern belagert, die von den umliegenden Bergen herabkamen. Johannes der Bucklige rettete ihn mit einem Entsatzheer. ${ }^{19}$ Im Jahr 497 gelang die Gefangennahme von Longinus von Cardala und der beiden Athenodoroi durch Johannes Skytha. Sie wurden in Konstantinopel unter großem Pomp enthauptet. ${ }^{20}$ Im isaurischen Antiocheia auf dem Berg Cragus hielten Longinus von Selinus und ein weiterer isaurischer Rebell namens Indes bis 498 aus. ${ }^{21}$ Schließlich wurden sie von Johannes dem Buckligen gefangen und nach Konstantinopel verbracht, wo sie enthauptet wurden. Anastasius ließ die isaurischen Wehrtürme zerstören. ${ }^{22}$ Die siegreichen Generäle bekamen den Konsulat; Johannes Skytha 498, Johannes der Bucklige im folgenden Jahr; die Überlebenden wurden nach Thrakien deportiert. ${ }^{23}$ Die dorthin in großer Zahl umgesiedelten Isaurier

16 PLRE II 688 s.n. 'Longinus 4'; Karamut / RUSSELl (1999) 364, 371 mutmaßen, daß er mit dem eradierten Namen nach dem comes in einer inschriftlichen Akklamation (unpubliziert, Museum Alanya) an Zeno in Nephelis gemeint sei.

17 Priscian, De laude Anastasii Z. 89; Mal. 393; PLRE 2, s.n. 'Diogenianus 4'.

18 Theoph. AM 5985.

19 Theoph. AM 5986.

20 Euagr. h.e. III 35; BrooKs (1893) 220.

21 Marc. com. a. 498; Euagr. h.e. III 35; Theoph. AM 5987f.; PLRE II 591 s.n. 'Indes'.

22 Theoph. AM 5988; Hopwood (1986).

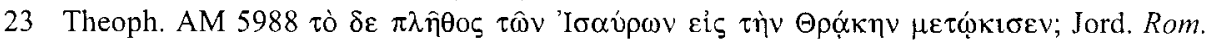
355 devicti et perquaquam exilio relegati urbesque eorum nonnullae solo usque prostratae; Priscian Pan. II 83 sic domuit post haec ne possint esse rebelles; vgl. SCHWARTZ (1934) 217. STEIN (1959) 84; HOPWOOD (1989a) 197. ELTON (2000a) 300. (2000b) 397 bezweifelt, daß es sich um einen Krieg gegen das ganze isaurische Volk gehandelt habe, es sei lediglich gegen isaurische Mächtige gegangen, auch sei nach dem Krieg nichts mehr gegen die Isaurier unternommen worden. Zum Beweis nennt er die hohe Zahl von Isauriern, die in Justinians Armee diente. Dies spricht jedoch nicht gegen einen vorhergehenden Krieg gegen alle Isaurier; abgesehen von der Frage nach dem Erkenntnisgewinn einer solchen Fragestellung hinsichtlich der Relevanz der isaurischen Ethnizität bei diesen Machtkämpfen. Daß mit der Geschichte des Candidus und den Isaurika ein plötzlicher Focus im Gegensatz zu ihrer früheren „obscurity“ in den Quellen gelegt worden sein soll, bringt ELTON (2000a) 298 zwar plausibel in den Zusammenhang mit Zenos Kaisertum und der nun erforderlichen Neubegründung des isaurischen Rufs. Angesichts ihrer zahlreichen Nennung in den erhaltenen Quellen vor dieser Zeit kann man jedoch keinesfalls von „obscurity“ sprechen. Allerdings dienten eine Reihe Römer bei den isaurischen Aufständi- 
lassen allerdings in den Quellen nichts mehr von sich hören. Vermutlich wollte Anastasius mit diesem Krieg eine konstantinopolitanischen Opposition gegen seine monophysitischen Neigungen (die er zu Beginn seiner Herrschaft allerdings noch nicht offen propagierte ${ }^{24}$ ) abwenden, um nicht das Schicksal des Basiliscus zu wiederholen. Der Krieg war ein willkommener Anlaß für den Kaiser, sich seines unliebsamen chalkedontreuen Patriarchen zu entledigen: Mitte 496 wurde Euphemius, der sich zum Chalkedonense bekannte und gerne die Gemeinschaft mit Rom wiederhergestellt hätte, wegen hochverräterischer Umtriebe mit den Führern des Aufstands (er hatte wohl nur in bester Absicht den Friedenswunsch des Anastasius verraten) vor ein klerikales Gericht gestellt, abgesetzt und deportiert. Diese Maßnahme war nicht gerade dazu angetan, die Spaltung mit Rom zu entschärfen. Als sein Nachfolger wurde der Presbyter Macedonius bestimmt mit der Auflage, Zenos Henotikon zu unterschreiben, das wegen seiner monophysitischen Tendenz Mißfallen in Rom erregt hatte. ${ }^{25}$

\subsection{Der Isaurierkrieg in der Panegyrik}

Das Epos Isaurika in sechs Büchern des Christodorus aus dem ägyptischen Koptos aus Anlaß des Sieges von Anastasius über die Isaurier 498 ist verloren. ${ }^{26}$ Dieses Werk gehörte zum Genre der „Patria“, Sammlungen von Topographien und Legenden einer bestimmten Gegend. ${ }^{27}$ Bezug auf die Isaurier nimmt er zudem in seiner erhaltenen Ekphrasis, einer Beschreibung der 80 Statuen in den Zeuxippos-Thermen, südlich der Hagia Sophia im Bereich des großen Palastes. Hier stellt Christodorus in seiner Beschreibung der Pompeius-Statue den siegreichen Kaiser in dessen Nachfolge. Schon Pompeius habe nämlich gegen die Isaurier gekämpft:

schen, Joh. Ant. frg. 214 b, dies würde aber eher gegen einen immerwährenden isaurischrömischen Konflikt nach SHAw sprechen; vgl. HeUCKE (1997) 52.

24 Anastasius war erst nach der Beendigung des Perserkrieges von 506/7 offen monophysitisch, auf Einfluß des Philoxenus: Theod. Lect. Epit. 466. 470. 472; Theoph. AM 5999; vgl. LUTHER (1997) 25.

25 Theod. lect. Epit. 449 = Theoph. AM 5987; Marc. com. s.a. 495 (falsch); PLRE II s.n. Ioannes 43; SCHWARTZ (1934) 222.

26 Suda X 525. BeCKBY AP (1957) I 184f.; Asmus (1913) 328; CAMERon (1965) 481; ThOMPSON (1946) 18; PorTMAnN (1988) 256, Anm. 4. Christodorus ist aus methodischen Gründen nicht aufgenommen bei KW. Asmus (1913) 328, Anm. 1 nennt noch Panolbius als einen Verfasser von „Isaurika“, doch dies wird im entsprechenden Suda-Artikel П 204 nicht erwähnt; vgl. PLRE II 829, s.n. 'Panolbius'.

27 Alexander KaZHDAN, s.v. 'Patria', ODB 1598. 
Darauf Pompeius, der Führer der mühegekrönten Ausonier, der, ein funkelndes $\mathrm{Mal}$ isauriermordender Stärke unter den Füßen zertrat die Schwerter isaurischer Männer kündend, daß er ins Joch der Knechtschaft gebeugt und mit Nikes unzerreißbarem Band gefesselt den Nacken des Taurus eben dieses der Mann, der allen ein leuchtendes Licht war und den heiligen Stamm Anastasius', des Kaisers, erzeugt hat. Denn dies zeigte der Welt mein Herr, der treffliche Herrscher, als er die Völker Isauriens mit seinen Waffen vernichtet. ${ }^{28}$

Die Isaurier werden also mit den kilikischen Piraten gleichgesetzt, was nicht der historischen Realität entspricht. ${ }^{29}$ Wo bei Christodorus Pompeius die isaurischen Piraten endgültig besiegt, wendet Priscian von Caesarea ${ }^{30}$ in seinem im Jahr 503 in lateinischer Sprache verfaßten Panegyricus im Vergleich zu Pompeius Überbietungstopik an; Anastasius übertreffe sogar Pompeius: Dieser habe es, obwohl „Eroberer der ganzen Welt", nicht geschafft, die Isaurier zu besiegen, Anastasius dagegen habe mit den Isauriern die semina belli überhaupt ausgerottet. ${ }^{31} \mathrm{Daß}$ er zuvor den Isauriersieg des P. Servilius Vatia erwähnt hat, zeigt den Vorrang des exemplaGebrauchs vor historischer Stringenz. ${ }^{32}$ Er stellt seinen Kaiser in Zeiten der Krise als ein Gottesgeschenk an das Reich dar: Die Isaurier werden von Gott mit Wahnsinn bestraft, weil sie es wagen, gegen den gottgesandten Kaiser zu rebellieren. Dieser Wahnsinn verleitet sie schließlich zum Krieg, und wegen der Frömmigkeit des Kaisers schickt Gott einen Sturm, der die Flotte der Isaurier zerstört. In ihrer Niederlage sehen sie ein, daß sie von Gott auf gerechte Weise bestraft wurden. Da von einem Seekrieg gegen die Isaurier in den historischen Quellen keine Rede ist, liegt hier ein

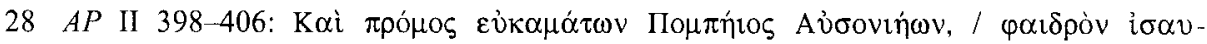

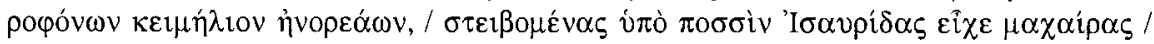

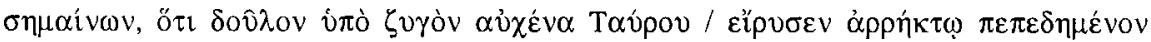

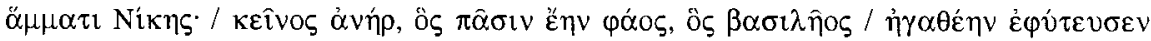

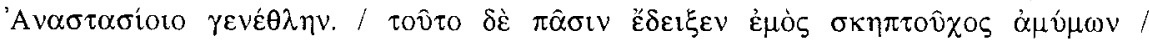

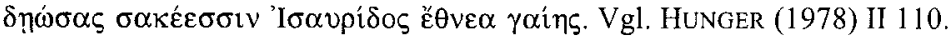

29 S.o. Kap. IV.3.2.5.

30 Lateinischer Grammatiker und Rhetor aus Caesarea in Mauretanien, lehrte um 500 in Konstantinopel, vgl. KW 293, 114; Chauvot Priscian (1986); Portmann (1988) 117 125.

31 Priscian Paneg. Z. 10-18, 82-86. Zur (von beiden Panegyrikern angesprochenen) Abstammung von Pompeius, die wohl kaum im engeren genealogischen Sinne zu verstehen ist, vgl. PORTMANN (1988) 118 gegen Johann OHLER, RE I 2 (1894) 2065: Priscian habe eine fiktive Genealogie erstellen wollen, so auch wieder Alan CAMERON, The House of Anastasius', GRBS 57 (1978), 260: die Wiederholung weise auf mehr als auf panegyrisches Kompliment hin, der Name Pompeius müsse tatsächlich in der Familie des Kaisers vorkommen, ebenso COYNE (1991) 78.

32 Priscian Paneg. Z. 80-86; vgl. PORTMAnN (1988) 118 f. 
Bezug zum Konflikt mit dem orthodoxen Goten Vitalian nahe, der mit seinen Truppen zur Zeit der Abfassung des Preisliedes die Hauptstadt ernsthaft bedrohte. ${ }^{33}$

Es war nach dem Handbuch des Menander Rhetor in der Panegyrik gebräuchlich, die Leiden der Bevölkerung unter dem Vorgänger hervorzuheben; dies sei besser, als den Vorgänger direkt zu attackieren. ${ }^{34}$ Es ist bemerkenswert, daß Priscian an dieser Stelle den Bogen bis in die mythische Vorzeit spannt (die einzige Anspielung auf heidnische Mythologie im Panegyricus), bis zum Kampf des Bellerophon gegen die Solymer, zwischen jenem gegen die Chimäre und die Amazonen. Von allen menschlichen Gegnern waren sie in der Schilderung Homers die stärksten:

Zum zweiten wieder kämpfte er mit den Solymern, den ruhmvollen;

Das war der härteste Kampf, sagte er, in den er je getaucht mit Männern. ${ }^{35}$

So wurde Bellerophontes' Sohn Isandros von Ares im Kampf gegen dieselben getötet. ${ }^{36}$ Die Panegyriken stilisieren die Isaurier zu gigantischen Reichsfeinden. Dies muß jedoch nicht bedeuten, daß der Kampf gegen sie als ein Kontinuum durch die gesamte Zeit des Imperiums hindurch angesehen wurde. Vielmehr scheint die Kaiserzeit gegenüber der Republik in der kollektiven Erinnerung verblaßt zu sein: In der spätantiken Panegyrik wurden eher Beispiele, exempla, aus der Mythologie und frühen Republik als aus der Kaiserzeit verwendet. ${ }^{37}$ Abgesehen davon, daß man auch in der Hohen Kaiserzeit nur auf möglichst frühe Zeiten rekurrierte, war diese nun für die spätantike Panegyrik gleichsam verbrannt, weil etliche der berühmten Kaiser wie Mark Aurel, die als Spender von exempla hätten dienen können, sich als Christenverfolger betätigt haben.

33 Priscian Paneg. Z. 38f. 89-97. 119-129; PLRE II 1171-1176 s.n. 'Fl. Vitalianus 2'. COYNE Priscian-Kommentar (1991) 8, 12 ff. plädiert für eine Spätdatierung auf Herbst 513. Der isaurische Krieg sei von Priscian lediglich als eine Folie benützt, um zeitgenössische Konflikte zu kommentieren, den Kampf gegen den rebellischen orthodoxen Goten Vitalian und den Westen (Papst Hormisdas und Theoderich), die den Goten unterstützten. Der Perserkrieg werde nur am Rande erwähnt, weil er als auswärtiger Konflikt von geringer Relevanz für die Vitalian-Krise sei.

34 Men. Rhet. 378, 20. Zur Panegyrik als Propaganda-Instrument vgl. CAMERon (1970); Sabine McCORMACK, 'Latin Prose Panegyrics: Tradition and Discontinuity in the Later Roman Empire' in: Revue des études augustiniennes 32 (1976), 21-77.

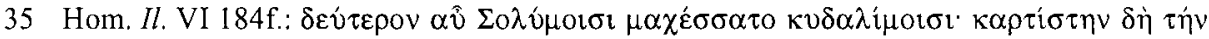

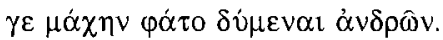

36 Hom. Il. VI 203f.; vgl. Od. V 283: Poseidon kommt von den Solymerbergen herab, um Odysseus zu versenken; Pindar Ol. XIII 63-92).

37 Vgl. H. W. Lichfield, 'National exempla virtutis in Roman Literature' in: Harvard Studies in Classical Philology 25 (1914), 1-71; PorTMANN (1988) 118, 218-222. Auch Johannes Lydus zeigt mehr Wissen über die Geschichte der Bürgerkriege der späten Republik als über jede andere Periode der römischen Geschichte, vgl. CARNEY (1971) zu Joh. Lyd. de mag. II 37. 
Prokop von Gaza in seiner Rede von 501 anläßlich der Aufstellung einer Statue für Anastasius verwendet ausschließlich exempla aus der griechischen und persischen Geschichte, wobei er diese sogar zuweilen kritisiert. So seien im Gegensatz zur brutalen Politik Philipps II. gegenüber den Griechen die Isaurier nach ihrer Niederlage milde behandelt worden. ${ }^{38} \mathrm{Er}$ setzt die Isaurier mit den Solymern synonym; historische Ereignisse werden nur dürftig erwähnt. ${ }^{39}$ Gemeinsam war Priscian und Procopius, daß sie die Isaurier als Fremde ansehen; Zeno als einen fremden Usurpator verdammen und die heidnischen Tendenzen bei den Isauriern betonen. ${ }^{40}$ Neben den genannten Panegyriken des Christodorus von Koptos, Priscian von Caesarea und Procopius von Gaza ist auf einer Inschrift über der Chalke, dem Torbau am Eingang des großen Kaiserpalastes, der Sieg über die Isaurier erwähnt. ${ }^{41}$

Der Sieg des Anastasius im Krieg gegen die Isaurier war in der byzantinischen Tradition offenbar so wirkungsmächtig, daß die Isaurier in den Perseusmythos Eingang gefunden haben, wie er in der Suda und bei Cedrenus wiedergegeben wird: nun waren eben nicht mehr die Kilikier, sondern die Isaurier die gefährlichsten Feinde der griechisch-römischen Zivilisation, denn sie werden hier sogar in Umkehrung der Chronologie vor den Kilikiern genannt. ${ }^{42}$

38 PortMANN (1988) 196.

39 Prokop verfaßte seine Panegyrik auf Anastasius zwischen 501 und 515: PLRE II, s. v. 'Procopius 8', 921f.; KW 293 Nr. 113; StEIN (1949) 77-106. 193. 204; CAPIZZI (1969) 303 Index s.v. Procopio di Gaza; zum Panegyricus PorTMAnN (1988) 195-198.

40 Vgl. Chauvot Christodonus / Procopius (1986).

41 AP IX 656; vgl. Cedr. I 563; Berger (1988) 243.

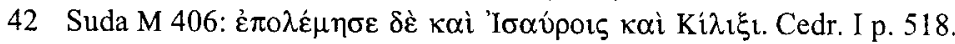

